\title{
Transiting exo-planets search for MOA-I data
}

\author{
A. Fukui ${ }^{1}$, F. Abe ${ }^{1}$, I. A. Bond ${ }^{2}$, K. Furusawa ${ }^{1}$, J. B. Hearnshow ${ }^{3}$, \\ Y. Itow ${ }^{1}$, K. Kamiya ${ }^{1}$, P. M. Kilmartin ${ }^{3}$, K. Masuda ${ }^{1}$, Y. Matsubara ${ }^{1}$, \\ N. Miyake ${ }^{1}$, Y. Muraki ${ }^{4}$ M. Nagaya ${ }^{1}$, S. Noda, K. Ohnishi ${ }^{5}$, \\ T. Saito ${ }^{6}$, T. Sako ${ }^{1}$, S. Sato ${ }^{7}$, D. J. Sullivan ${ }^{8}$, T. Sumi ${ }^{1}$, \\ P. J. Tristram ${ }^{3}$, T. Yanagisawa ${ }^{9}$, and P. C. M. Yock ${ }^{10}$ \\ ${ }^{1}$ Solar-Terrestrial Environment Laboratory, Nagoya University, Nagoya 464-8601, Japan \\ email: afukui@stelab.nagoya-u.ac.jp \\ ${ }^{2}$ Institute for Information and Mathematical Sciences, Auckland, New Zealand \\ ${ }^{3}$ Department of Physics and Astronomy, University of Canterbury, Christchurch, New Zealand \\ ${ }^{4}$ Department of Physics, Konan University, Kobe, Japan \\ ${ }^{5}$ Nagano National College of Technology, Nagano, Japan \\ ${ }^{6}$ Tokyo Metropolitan College of Aeronautics, Tokyo, Japan \\ ${ }^{7}$ Department of Physics and Astrophysics, Faculty of Science, Nagoya University, Japan \\ ${ }^{8}$ School of Chemical and Physical Sciences, Victoria University, Wellington, New Zealand \\ ${ }^{9}$ Advanced Space Technology Research Group, Institute of Aerospace Technology, Japan \\ Aerospace Exploration Agency (JAXA), Japan \\ ${ }^{10}$ Department of Physics, University of Auckland, Auckland, New Zealand
}

\begin{abstract}
The Microlensing Observations in Astrophysics (MOA) is a microlensing survey conducted at Mt. John Observatory in New Zealand. We searched transiting planet candidates from the MOA-I Galactic bulge data, which have been obtained with a $61 \mathrm{~cm} \mathrm{B \& C}$ telescope from 2000 to 2005 for a microlensing search. Although this survey data were dedicated to microlensing, they are also quite useful for searching transiting objects because of the large number of stars monitored ( $\sim 7$ million) and the long span of the survey ( $\sim 6$ years). From our analysis, we found 58 transiting planet candidates. We are planning to follow up these candidates with high-precision spectroscopic and photometric observations for further selection, toward the detection of planets by radial velocity observations.
\end{abstract}

\section{Introduction}

Although about 50 transiting extra-solar planets have been discovered so far, it is still important to increase this number (see other contributions in this volume). Another important technique for detecting planetary systems is microlensing. This technique is sensitive to low-mass planets at separations of a few AU (Gaudi 2007), which is complementary to other techniques such as the radial velocity method and the transit method. The two observational techniques of microlensing and transit are similar in requiring continuous photometric surveys for large number of stars. Thus, it is worthwhile to search for transiting planets in microlensing survey archive data. The opposite is difficult because microlensing events occur only once and instantaneous follow-up observations are needed to confirm the planetary nature.

The Microlensing Observations in Astrophysics (MOA) is a microlensing survey groups conducted at Mt. John Observatory in the south island of New Zealand, and currently achieving some excellent results using the 1.8-m MOA-II telescope (e.g. Bennett et al. 2008). We searched for transiting planet candidates in the MOA-I Galactic bulge archive 
data, which have been obtained from 2000 to 2005 with the $61-\mathrm{cm}$ B\&C telescope for the microlensing survey. We show our observations, analysis, results, and future plan.

\section{Observation}

MOA-I data have been taken by using the 61-cm B\&C telescope with three 2048 pix $\times 4096$ pix CCDs camera MOA-cam2 (Fig. 3), which have a field-of-view of $\sim 1.3 \mathrm{deg}^{2}$. We have a total of 16 fields $\left(\sim 20 d e g^{2}\right)$ toward the Galactic Bulge and each field has an average sampling rate of $\sim 1$ to 5 times per night, depending on the field. These data had been analyzed in near realtime, and when an interesting (i.e. anomalous or high magnification) microlensing event was detected, the corresponding field had been observed with higher sampling. A total of 1000 - 2800 images in each field had been taken across 6 years.

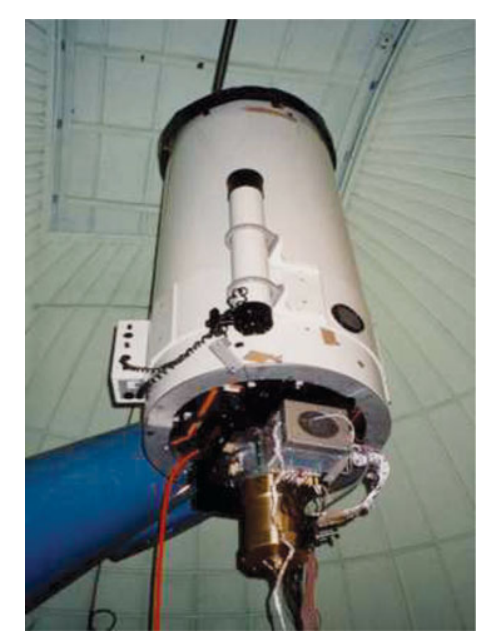

Figure 1. The Boller and Chivens $(B \& C)$ telescope with the CCD camera MOA-cam2. The telescope has a $61-\mathrm{cm}$ aperture Richey-Chretien Cassegrain reflector and the camera has three $2048 \times 4096$ pixels SITe thinned back-illuminated CCD chips (ST-002A).

\section{Analysis}

We applied the Difference Image Analysis (DIA), which we usually use for the microlensing analysis for the photometry of crowded fields such as the Galactic Bulge, for the analysis of transits. After first reduction (dark subtraction and flat correction) in a standard manner, each observing image was subtracted to a reference image which was chosen as the best image (good seeing, high Signal-to Noise ratio, and low airmass) and was convolved to match the seeing and the scale of the observed image. Then we measured the brightnesses of all stars, about 2.3 millon stars detected on the reference images of one of 3 CCD chips (chip2, which has less noise), by using the empirical PSF fitting. As a result, we got the light curves of these stars with the photometric precisions shown in Fig. 2.

We selected about $3.2 \times 10^{5}$ objects which have photometric precisions better than $2.0 \%$. Then we used the Box-fitting Least Square (BLS) algorithm (Kovacs, Zucker, \& Mazeh 2002) to search for periodicities in these objects. The searching range was from 0.4 to 25 days. The period range affected by alias effects due to the earth's rotation were 


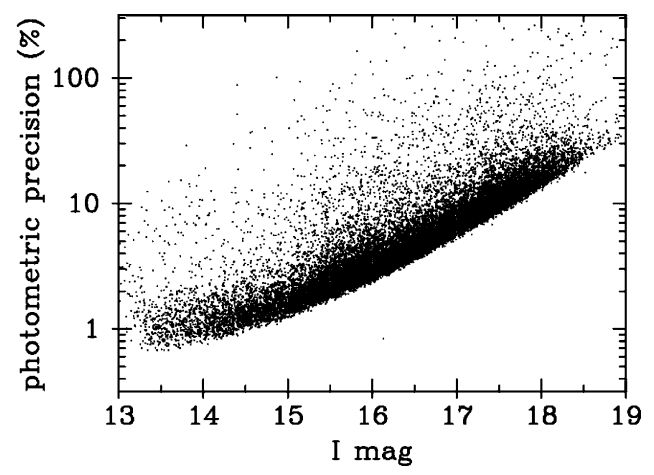

Figure 2. Distribution of the photometric precisions as function of $I$ magnitude in a field. We selected objects which have better precisions than $2.0 \%$.

masked. We calculated the $\mathrm{S} / \mathrm{N}$ of each period from the $\Delta \chi^{2}$, which is the difference of the $\chi^{2} \mathrm{~s}$ between the box-like fit and the constant fit for a phase-folded light curve. Next we adopted the selection criteria $\mathrm{S} / \mathrm{N}>7$. After that, we fitted a transit model to the selected light curves by minimum- $\chi^{2}$ and picked out about 1,000 transit-like candidates. A criteria for this selection was decided by comparing the distributions of the $\chi^{2} \mathrm{~s}$ of non-transit light curves to that of artificial transit light curves.

Finally, we selected the final candidates by the careful screening of the objects which show secondary eclipses or tidal effects in their light curves, both of which are clear signals of low-mass star companions.

\section{Results and Future plan}

Finally we detected 58 new transiting objects (Fig. 3 red dots) from the MOA-I data in this analysis, in addition to the previous work (Abe et al. (2005), Fig. 3 black dots) which analyzed the first 3 years' MOA-I data. 18 of these candidates have transiting depths of less than $2.6 \%$, which is the largest value among the known transiting planets, and they have a higher possibility of being real planets. An example of light curve is shown in Fig. 4.

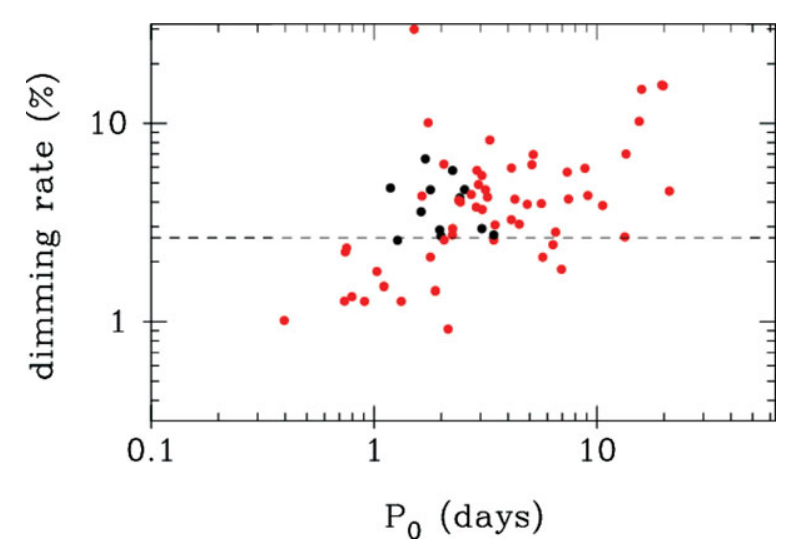

Figure 3. Depth and period of 58 new transiting objects (red dots) additional to the previous work (Abe et al. (2005), black dots). The dashed line shows the maximum value of the depth of transit among the known transiting planets. 
These candidates would still include many false positives caused by low-mass eclipsing binary stars, because the radii of giant planet and low mass star are almost the same and the blending effect can dilute the depth of transit (Konacki et al. 2003). So we need to confirm the nature of these candidates by measuring radial velocities. However, our candidates are all in crowded fields and are relatively faint, so that long observing times with an 8-m class telescope are needed. Thus, we want to eliminate as many contaminants as possible from our current candidates before the radial velocity measurements.

We are planning to observe our candidates with the 8.2-m Subaru telescope in Hawaii with the High-Dispersion Spectrograph (HDS) to eliminate the spectroscopic binaries, and with the 1.4-m Infra Red Survey Facility (IRSF) telescope in South Africa to screen transits showing a deep secondary eclipse. We aim to detect real planets in the future with spectroscopic observations.

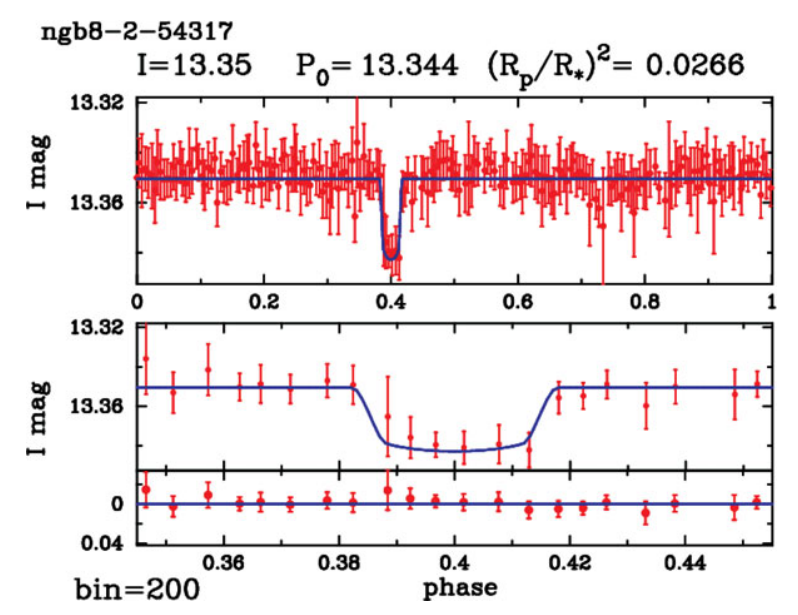

Figure 4. One of light curves for our transit candidates. The upper panel shows the entire phase-folded light curve and the middle panel zooms on the transit. Solid lines show the best-fit transit model and the lower panel shows the residuals. Data points are binned by 200 .

\section{References}

Abe, F., et al. 2005, MNRAS, 364, 325

Bennett, D. P., et al. 2008, ApJ, accepted

Charbonneau, D., Brown, T. M., Burrows, A., \& Laughlin, G. 2007, Protostars and Planets V, B. Reipurth et al. (eds.), University of Arizona Press, Tucson, 701

Gaudi, B. S. 2007, "Extreme Solar Systems", ASP Conference Series, ed. Debra Fischer, Fred Rasio, Steve Thorsett and Alex Wolszczan

Konacki, M., Torres, G., Jha, S., and Sasselov, D., D 2003, Nature, 421

Kov́acs, G., Zucker, S., \& Mazeh, T. 2002, A\&AA, 391, 369 